\title{
Intracranial Arteriovenous Malformation-Solitary Vascular Lesion: A Case Report
}

\author{
Amandeep $\operatorname{Singh}^{1}{ }^{\circ}$, Aaina Devgan ${ }^{2}$, Sumanjeet Kaur ${ }^{3}{ }^{\circ}$
}

\begin{abstract}
Brain arteriovenous malformations (AVMs) are an abnormal vascular web that comprises the nidus, feeding arteries, and draining veins. The frequently used grading system is Spetzler-Martin grading. The majority of the brain AVMs are usually asymptomatic, but the common presentation can be headache, seizure, intracerebral hemorrhage, or focal neurological deficit. A 27-year-old male presented with the chief complaint of headache with episodes of ataxia over the course of 4-5 months. Magnetic resonance imaging (MRI) findings revealed multiple tortuous vessels with central nidus in the right posteroinferior cerebellar hemisphere. Also on contrast-enhanced computed tomography (CECT) brain angiography, area of the dilated tuft of vessels in the right posteroinferior cerebellar hemisphere with a nidus of vessels within brain parenchyma with arterial feeders and draining veins was observed. Computerized tomography (CT) of the brain angiography is a modality of choice for the depiction of feeding arteries and draining veins.

Keywords: Brain arteriovenous malformation, Nidus, Tortuous vascular channels.

AMEI's Current Trends in Diagnosis \& Treatment (2021): 10.5005/jp-journals-10055-0122
\end{abstract}

\section{INTRODUCTION}

Brain arteriovenous malformations (AVMs) are described as abnormal vascular channels within the brain parenchyma. Vascular lesions of the brain are not so common lesions, so they act as a diagnostic challenge because of their alike imaging features and clinical manifestations. ${ }^{1}$

A brain AVM comprises the complex abnormal dilated tortuous vascular channels referred to as nidus. As nidus deficits a capillary bed, blood from supplying arteries is straightforwardly shunted into the draining veins. ${ }^{2}$ Brain AVM might sometimes coexist with other vascular disorders, such as moya moya disease or hereditary hemorrhagic telangectasia. ${ }^{3}$

Also when a brain AVM is detected, precise details regarding the natural risk posed by a brain AVM and the risks related to the treatment need to be reported. ${ }^{1}$

Evaluation and treatment of brain AVM is frequently a multidisciplinary approach involving neurosurgeons, neurologists, neuroradiologists, radiotherapists, and numerous other medical specialists. $^{3}$

\section{Case Description}

A 27-year-oldmale presented with the chief complaint of headache that was diffuse in nature and of mild to moderate intensity with episodes of ataxia over the course of 4-5 months. He otherwise was normotensive and afebrile, and the pulse rate measured was $72 \mathrm{bpm}$ at the time of general physical examination. There was no focal neurological deficit, and also there was no history of seizures. On magnetic resonance imaging (MRI), $\mathrm{T} 1$ and $\mathrm{T} 2 \mathrm{WI}$ show multiple dilated tortuous vessels with central nidus in relation to the right posteroinferior cerebellar hemisphere (likely non-eloquent cortex) (Fig. 1). Nidus measures approximately $2.5 \times 2 \times 1.7 \mathrm{~cm}$ in size. Ectasia of draining and feeding vessels was seen. No obvious hemorrhage was noted. The patient was further advised contrast-enhanced computed tomography (CECT) angiography for better delineation of arterial feeders and draining veins. \begin{tabular}{l}
\hline${ }^{1-3}$ Department of Radiodiagnosis and Imaging, Sri Guru Ram Das \\
Institute of Medical Sciences and Research, Amritsar, Punjab, India
\end{tabular}

Corresponding Author: Amandeep Singh, Department of Radiodiagnosis and Imaging, Sri Guru Ram Das Institute of Medical Sciences and Research, Amritsar, Punjab, India, Phone:+91 09872454954, e-mail:dr.amancs@gmail.com

How to cite this article: Singh A, Devgan A, Kaur S. Intracranial Arteriovenous Malformation-Solitary Vascular Lesion: A Case Report. AMEI's Curr Trends Diagn Treat 2021;5(1):55-57.

Source of support: Nil

Conflict of interest: None

On CECT brain angiography, there was an area of a dilated tuft of vessels in the right posteroinferior cerebellar hemisphere with a nidus of vessels within brain parenchyma with arterial feeders and draining veins (Fig. 2), finding consistent with MRI. Associated mild gliotic changes are also observed. Arterial feeders are seen arising from bilateral posteroinferior cerebellar artery and multiple dilated tortuous veins, which are seen in vermis that was seen draining into the great cerebellar vein and left sigmoid sinus. The patient was treated with corticosteroids, and symptoms were almost resolved.

\section{Discussion}

Brain AVMs constitute a rare disease with a prevalence of $0.06-0.11 \%$ and an incidence of $0.01-0.0013 \%$. The global annual mortality and severe morbidity associated with AVM have been estimated to be 1 and 1.5\%, respectively, and increase with repeated hemorrhage. ${ }^{4}$ Brain AVMs are congenital lesions, but patients use to present later, most frequently with intracranial bleed or seizures. ${ }^{1}$

Brain AVMs are anomalous connections between arteries that normally supply the brain tissue and veins that normally drain the 

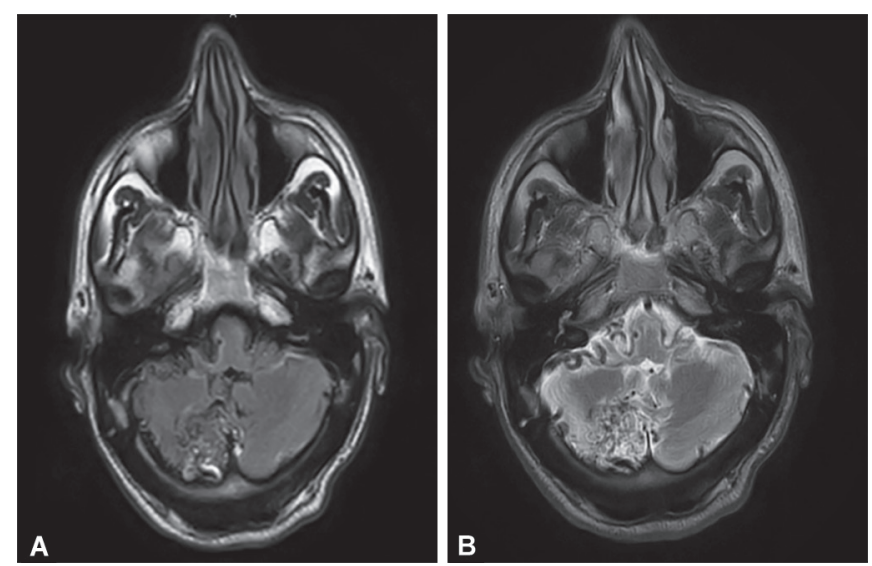

Figs 1A and B: Axial T1 WI (A) and axial T2 WI (B) show multiple dilated tortuous vessels with central nidus seen in relation to right posteroinferior cerebellar hemisphere causing indentation over the cerebellar tonsil with a mild deviation of vermis
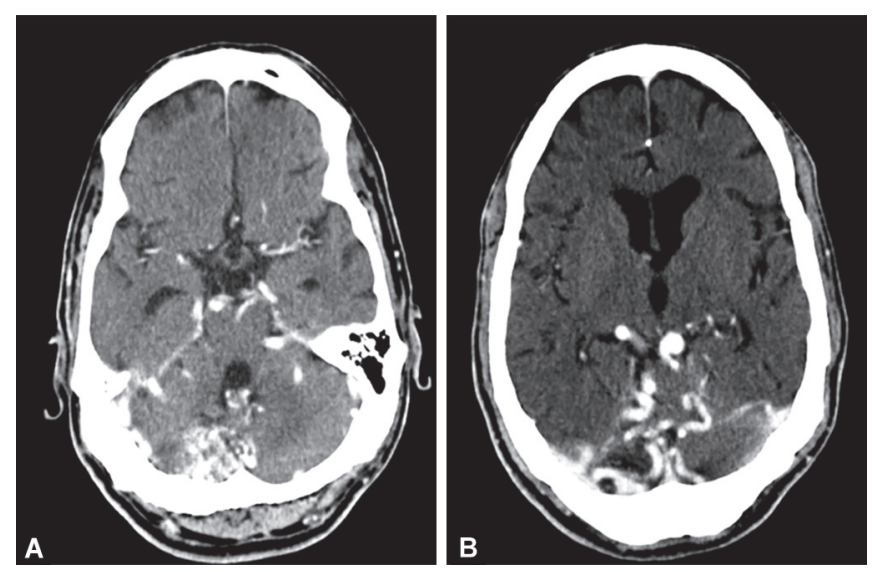

Figs 2A and B: (A) Axial CT angiography shows tuft of vessels in relation to posteroinferior aspect of the right cerebellum; (B) Axial CT angiography shows dilated feeding and draining vascular channels

brain, thus resulting in arteriovenous shunting. There is an absence of a true capillary bed.

The transition between supplying arteries and draining veins takes place through a nidus, that is, abnormally intertwined vessels positioned in the brain parenchyma. ${ }^{1}$ The vascular apparatuses of the nidus range from well-differentiated arteries and veins to dysplastic vascular channels. Gliotic or the nonfunctional tissue is commonly found interposed within the AVM and in the perinodal region. ${ }^{2}$ Calcification may be present in this gliotic tissue. The nidus can additionally be depicted as glomerular (intervening brain is not normal) or diffuse (normal intervening brain is present). Glomerular is the most frequently out of the two. ${ }^{5}$

The existence of the isolated nidus with intervening gliotic brain tissue aids to differentiate classic brain AVM from the rest of the intracranial arteriovenous shunting lesions, such as cerebral proliferative angiopathy, pial arteriovenous fistula, dural arteriovenous fistula, and venous-predominant AVM. ${ }^{2}$

Brain AVMs can happen anywhere in the brain or in the spinal cord, but the majority of them favors supratentorial location. Ninety-eight percent of these lesions are solitary. ${ }^{5}$
Intracerebral hemorrhage is the most severe and common presentation or complication of brain AVMs. The bleeding can be intraparenchymal and/or intraventricular, which corresponds to a breach within the nidus or sometimes in a tortuous draining vein. ${ }^{6}$ Additional symptoms like headache, seizure, and transient ischemic attacks have also been described. ${ }^{4}$

The Spetzler-Martin grading scale system is a commonly used scale for categorizing the AVMs, comprising three parameters that include the size of the lesion, the pattern of venous drainage (superficial or deep), and neurological eloquence of the surrounding brain. It is broadly used for accessing the risk of surgery and a consensus therapeutic guideline in adult patients. $^{7}$

The diagnosis of intracranial AVMs essentially depends on the cross-sectional imaging and the dynamic 4D sequences, both in the complicated AVMs, such as those associated with intracranial bleed, and in the unruptured AVM. ${ }^{6}$

The most commonly used imaging modality used for the diagnosis of brain AVMs is a computed tomography (CT). In addition, MRI scan has a unique sensitivity in detecting the remote hemorrhage, and conventional catheter cerebral angiography constantly remained a gold standard in the identification of brain AVMs. ${ }^{8}$ The diagnostic criteria include the presence of a nidus seen embedded within the brain tissue and early venous drainage, which can be best seen on the dynamic studies, the standard of reference being conventional catheter angiography. But nowadays, imaging modalities like dynamic studies, such as dynamic MR and CT angiography, are progressively being used in the detection of early drainage for tiny lesions and consequent benefit in establishing the diagnosis. ${ }^{1}$

Likewise in our case, on T1 and T2 WI, there were multiple tortuous vessels with central nidus seen in relation to the right posteroinferior cerebellar hemisphere (likely non-eloquent cortex) causing indentation over the cerebellar tonsil with a mild deviation of the vermis. Nidus measures approximately $2.5 \times 2 \times 1.7 \mathrm{~cm}$ in size. Ectasia of feeding arteries and draining veins was observed. No obvious areas of hemorrhage were noted (Fig. 1). On CECT brain angiography, the dilated tuft of vessels were observed in the right posteroinferior cerebellar hemisphere with central nidus along with arterial feeders and draining veins (Fig. 2). Arterial feeders were seen arising from the bilateral posteroinferior cerebellar artery, and multiple dilated tortuous veins were seen draining into the great cerebellar vein and left sigmoid sinus.

Management choices of brain AVM are increasingly made by multidisciplinary squads. Radiologists play a crucial role in the identification, grading, and treatment of brain AVMs. So, a detailed understanding of these lesions is necessary for guiding patient management. ${ }^{2}$ The medical therapies include anticonvulsants and steroids. While taking a therapeutic decision, it is of utmost essential to consider the patient's age, location, deep venous drainage, size of AVMs, and the existence of the associated aneurysms. If any large aneurysm is encountered, it must be secured before any interventions. $^{8}$

However, as intracranial hemorrhage is the most dangerous complication of AVM, so keeping the complication in view, treatment should be aimed at complete occlusion, or if possible, total removal is to be considered in order to eliminate the risk of future hemorrhage. Endovascular embolization is increasingly used to treat intracerebral AVMs. ${ }^{4}$ 


\section{Conclusion}

Brain AVMs are said to be congenital in nature with direct arteriovenous shunting without any intervening capillary bed. The patient is usually asymptomatic until a large aneurysm ruptured to cause intracerebral hemorrhage, despite of that patient can present with chronic headache, seizures, and focal neurological deficits but later in life. Dynamic CT or MRI angiography is increasingly used nowadays for the identification and early diagnosis of AVMs.

\section{OrCID}

Amandeep Singh (10 https://orcid.org/0000-0003-0656-3368

Aaina Devgan (1) https://orcid.org/0000-0001-5994-862X

Sumanjeet Kaur (1) https://orcid.org/0000-0003-3290-2909

\section{References}

1. Geibprasert S, Pongpech S, Jiarakongmun P, et al. Radiologic assessment of brain arteriovenous malformations: what clinicians need to know. Radiographics 2010;30(2):483-501. DOI: 10.1148/ rg.302095728.

2. Tranvinh E, Heit JJ, Bey LH, et al. Contemporary imaging of cerebral arteriovenous malformations. Am J Roentgenol 2017;208(6):1320-1330. DOI: 10.2214/AJR.16.17306
3. Joint Writing Group of the Technology Assessment Committee American Society of Interventional and Therapeutic Neuroradiology; Joint Section on Cerebrovascular Neurosurgery a Section of the American Association of Neurological Surgeons and Congress of Neurological Surgeons; Section of Stroke and the Section of Interventional Neurology of the American Academy of Neurology, et al. Reporting terminology for brain arteriovenous malformation clinical and radiographic features for use in clinical trials. Stroke 2001;32(6):1430-1442. DOI: 10.1161/01.str.32.6.1430.

4. Cronqvist $M$, Wirestam R, Ramgren $B$, et al. Endovascular treatment of intracerebral arteriovenous malformations: procedural safety, complications, and results evaluated by MR imaging, including diffusion and perfusion imaging. Larsson Am J Neuroradiol 2006;27(1):162-176. PMID: 16418378, PMCID: PMC7976081.

5. Smith $A B$. Vascular malformations of the brain: radiologic and pathologic correlation. J Am Osteopath Coll Radiol 2012;1(1):10-22. Available at: https://www.jaocr.org/articles/vascular-malformationsof-the-brain-radiologic-and-pathologic-correlation

6. Barreau X, Marnat G, Gariel F, et al. Intracranial arteriovenous malformations Diagn Interv Imaging 2014;95(12):1175-1186. DOI: 10.1016/j.diii.2014.10.004.

7. Lin HC, Huang WY, Chen SJ, et al. Whether the Spetzler-Martin grading scale is adequate to children with intracranial arteriovenous malformations? J Med Sci 2014;34(3):104-109. DOI: 10.4103/10114564.134375 .

8. Shaikh N, Al Kubaisi A, Mohsin Khan M, et al. Cerebral arteriovenous malformation from classification to the management. In: Vascular malformations of the central nervous system. 2020. 